\title{
Hahn-Banach theorems and subgradients of set-valued maps ${ }^{* 1}$
}

Jian Wen Peng ${ }^{1}$, Heung Wing Joseph Lee ${ }^{2}$, Wei Dong Rong ${ }^{3}$, Xin Min Yang ${ }^{4}$

${ }^{1}$ College of Mathematics and Computer Science, Chongqing Normal University, Chongqing 400047, P. R. China; Department of Mathematics, Inner Mongolia University, Hohhot 010021, Inner Mongolia, P. R. China.email:pjw7503_cn@sina.com

2 Department of Applied Mathematics, The Hong Kong Polytechnic University, Hung Hom, Kowloon, Hong Kong.

${ }^{3}$ Department of Mathematics, Inner Mongolia University, Hohhot 010021, Inner Mongolia, P. R. China.

${ }^{4}$ College of Mathematics and Computer Science, Chongqing Normal University, Chongqing 400047, P. R. China.

Abstract. Some new results which generalize the Hahn-Banach theorem from scalar or vector-valued case to set-valued case are obtained. The existence of the Borwein-strong subgradient and Yang-weak subgradient for set-valued maps are also proven. we present a new Lagrange multiplier theorem and a new Sandwich theorem for set-valued maps.

Key Words: Hahn-Banach theorem, set-valued map, subgradient, Lagrange multiplier theorem, Sandwich theorem

Mathematics Subject Classification: 54C60, 54C20, 49J52.

\section{Introduction}

A very important result in functional analysis about the extension of a linear functional dominated by a sublinear function defined on a real vector space was first presented by Hahn [1] and Banach [2] and it is stated as follows:

Theorem 1.1 (Hahn-Banach Theorem) Let $X$ be a real linear space and let $p$ be a real-valued sublinear function defined on $X$ satisfying the conditions:

$$
\begin{aligned}
& p(x+y) \leq p(x)+p(y) \\
& p(\alpha x)=\alpha p(x), \text { for all } \alpha \geq 0 .
\end{aligned}
$$

\footnotetext{
${ }^{1 *}$ This research was partially supported by the National Natural Science Foundation of China (Grant No. 10171118 and Grant No. 10261005) and Education Committee project Research Foundation of Chongqing (Grant No. 030801), and the research Committee of the Hong Kong Polytechnic University.
} 
Let $X_{0}$ be a real linear subspace of $X$ and $f_{0}$ a real-valued linear functional defined on $X_{0}$, i.e.

$$
f_{0}(\alpha x+\beta y)=\alpha f_{0}(x)+\beta f_{0}(y) \quad \text { for all } x, y \in X_{0} \text { and real } \alpha, \beta .
$$

Let $f_{0}$ satisfy $f_{0}(x) \leq p(x)$ on $X_{0}$. Then there exists a real-valued linear functional $F$ defined on $X$ such that i) $F$ is an extension of $f_{0}$, i.e., $F(x)=f_{0}(x)$ for all $x \in X_{0}$, and ii) $F(x) \leq p(x)$ on $X$.

The complex version of Theorem 1.1 was proved by Bohnenblust and Sobczyk in [3].

Generalizations and variants of the Hahn-Banach theorem were developed in different directions in the past. Weston [4] generalized Theorem 1.1 as follows:

Theorem 1.2 Let $X$ be a real linear space and let $g(x)$ be a real-valued convex function defined on $X$, i.e.

$$
g(\lambda x+(1-\lambda) y) \leq \lambda g(x)+(1-\lambda) g(y), \forall x, y \in X, \forall \lambda \in[0,1]
$$

Let $X_{0}$ be a real linear subspace of $X$ and $f_{0}$ a real-valued linear functional defined on $X_{0}$. Let $f_{0}$ satisfy $f_{0}(x) \leq g(x)$ on $X_{0}$. Then there exists a real-valued linear functional $F$ defined on $X$ such that i) $F$ is an extension of $f_{0}$, i.e., $F(x)=f_{0}(x)$ for all $x \in X_{0}$, and ii) $F(x) \leq g(x)$ on $X$.

Hirano, Komiya and Takahashi [5] proved a Hahn-Banach theorem in which a concave functional is dominated by a sublinear functional in a nonempty convex set. Day [7], Zowe [8-10], Elster and Nehse [11], Wang [12] and Shi [13] generalized the Hahn-Banach theorem to the partially ordered linear space. Chen and Craven [14] presented a Hahn-Banach theorem in which a continuous vector-valued affine map is dominated by a $K$-convex vectorvalued map as follows:

Theorem 1.3 (Theorem 2 in [14]) Let $X$ be a real linear topological space and let $(Y, K)$ be a real order-complete linear topological space, with order cone $K$. Let $C \subset X$ be convex, with int $\not \neq \emptyset$. Let the vector-valued map $f: C \rightarrow Y$ be $K$-convex, i.e.

$$
\lambda f(x)+(1-\lambda) f(y) \in f(\lambda x+(1-\lambda) y)+K, \forall x, y \in X, \forall \lambda \in[0,1]
$$

Let $X_{0}$ be a proper subspace of $X$, with $X_{0} \cap$ core $C \neq \emptyset$. Let $h: X_{0} \rightarrow Y$ be a continuous affine map such that $f(x)-h(x) \in K$ for each $x \in X_{0} \cap C$. Then there exists a continuous affine map $l: X \rightarrow Y$ such that i) $l$ is an extension of $h$, i.e., $l(x)=h(x)$ for all $x \in X_{0}$, and ii) $f(x)-l(x) \in K$ for all $x \in C$.

Yang [15] proved a Hahn-Banach theorem in which a linear map is weakly dominated by a set-valued map which is convex. Meng [16] obtained Hahn-Banach theorems by using 
concept of efficient for $K$-convex set-valued maps. The various geometric forms of HahnBanach theorems (i.e., Hahn-Banach separation theorems) was presented by Eidelheit [17], Rockafellar [18], Deumlich, Elster and Nehse [19], Taylor and Lay [20], Wang [12], Shi [13], Elster and Nehse [37] in different spaces.

Hahn-Banach theorems plays a central role in functional analysis, convex analysis and optimization theory. For more details on Hahn-Banach theorems as well as their applications, please also refer Jahn [6] and [21], Kantorovitch [22], Lassonde [23], Rudin [24], Schechter [25], Aubin and Ekeland [26], Yosida [32] and the references therein.

In the second section of this paper we collect some basic definitions and preliminary facts needed later.

In the third section of this paper we derive some new and interesting Hahn-Banach theorems for set-valued maps

In the fourth section of this paper we prove the existence of two kinds of subgradients of set-valued maps.

In the fifth section of this paper we prove a new Lagrange multiplier theorem for setvalued maps.

In the sixth section of this paper we prove a new Sandwich theorem for set-valued maps.

\section{Preliminaries}

Throughout this paper, let $Y$ be a real linear topological space, let $K \subset Y$ be a pointed closed convex cone. The partial order $\leq$ on a partially ordered linear space $(Y, K)$ is defined by $y_{1}, y_{2} \in Y, y_{1} \leq y_{2} \Leftrightarrow y_{2}-y_{1} \in K$. If $K$ has a nonempty interior (int $K$ ), then a weak ordering in $\mathrm{Y}$ is also defined by $y_{1}, y_{2} \in Y, y_{1} \ngtr y_{2} \Leftrightarrow y_{1}-y_{2} \notin i n t K \Leftrightarrow y_{2}-y_{1} \notin-i n t K$. Let $X$ be a real linear space, the algebraic interior of $C \subset X$ is defined by:

$$
\text { core } C=\left\{x \in C \mid \forall x_{1} \in X, \exists \delta>0, \text { s.t. } \forall \lambda \in(0, \delta), x+\lambda x_{1} \in C\right\}
$$

If $A$ and $B$ are two sets, then $A-B$ denotes the set $\{a-b \mid a \in A, b \in B\}$ and $A-b$ denotes the set $\{a-b \mid a \in A\}$. By $L(X, Y)$ we denote the space of linear operators from $X$ into $Y$.

We recall some necessary definitions as follows.

Definition 2.1([29]). A topological vector space $Y$, partially ordered by a convex and pointed cone $K$, is order-complete if every subset $M$ which has an upper bound $b$ in terms of the ordering (i.e., $(\forall y \in M) b-y \in K)$ has a supremum $\hat{b}$ (i.e., there exists $\hat{b} \in Y$ such that $\hat{b}$ is an upper bound to $M$, and each upper bound $b$ to $M$ satisfies $b-\hat{b} \in K)$. 
Remark 2.1. Let $(Y, K)$ be an order-complete topological vector space, then, every subset $M$ which has a lower bound $d$ in terms of the ordering (i.e., $(\forall y \in M) y-d \in K$ ) has a infimum $\hat{d}$ (i.e., there exists $\hat{d} \in Y$ such that $\hat{d}$ is a lower bound to $M$, and each lower bound $d$ to $M$ satisfies $\hat{d}-d \in K)$.

Definition 2.2([27, 30]). Let $C \subset X$ be a convex set. A set-valued map $F: C \rightarrow 2^{Y}$ is called $K$-convex on $C$, if for every $x, y \in C$ and $\lambda \in(0,1)$

$$
\lambda F(x)+(1-\lambda) F(y) \subset F(\lambda x+(1-\lambda) y)+K .
$$

Remark 2.2. By Proposition 2.2.3 in [30], a set-valued map $F: C \rightarrow 2^{Y}$ is $K$-convex on $C$ if and only if Epi $F=\{(x, y) \mid x \in C, y \in Y, y \in F(x)+K\}$ is a convex set. From [15], we know that a set-valued map $F: C \rightarrow 2^{Y}$ is convex if $\operatorname{Gr} F=\{(x, y) \mid x \in C, y \in Y, y \in F(x)\}$ is a convex set.

Definition 2.3([26]). Let $C \subset X$ be a nonempty set. $F$ is called a strict set-valued map from $C$ to $Y$, if the images $F(x)$ are nonempty for all $x \in C$.

\section{Hahn-Banach theorems of set-valued maps}

In this section, a new Hahn-Banach theorem in which an affine map is dominated by a $K$-convex set-valued map is first proven. Then some meaningful results which are based on this new result are also shown.

Theorem 3.1. $\quad$ Let $X$ be a real linear space, and let $(Y, K)$ be a real order-complete linear topological space. Let $F: C \rightarrow 2^{Y}$ be a strictly $K$-convex set-valued map, where $C \subset X$ is a convex set. Let $X_{0}$ be a real linear proper subspace of $X$, with $X_{0} \cap \operatorname{core} C \neq \emptyset$ and $h: X_{0} \rightarrow Y$ be an affine map satisfying $F(x)-h(x) \subset K$ for all $x \in X_{0} \cap C$. Then there exists an affine map $l: X \rightarrow Y$ such that i) $l$ is an extension of $h$. i.e., $l(x)=h(x)$ for all $x \in X_{0}$, and ii) $F(x)-l(x) \subset K$ for all $x \in C$.

Proof. The theorem holds trivially if $C=X_{0}$. Assume that $C \neq X_{0}$. Since $X_{0}$ be a proper subspace of $X$, there exists $x_{0} \in X \backslash X_{0}$. Let

$$
X_{1}=\left\{x+r x_{0}: x \in X_{0}, r \in R\right\} .
$$

It is clear that $X_{1}$ be a subspace of $X, X_{0} \subset X_{1}, X_{1} \cap$ core $C \neq \emptyset$ and the above representation of $x_{1} \in X_{1}$ in the form $x_{1}=x+r x_{0}$ is unique. Note that for each point $\hat{x} \in X_{0} \cap$ core $C$, if $|k|$ is sufficiently small, then $\hat{x} \pm k x_{0} \in X_{1} \cap C$. For arbitrary $x_{1}, x_{2} \in X_{0} \cap$ core $C$, if $x_{1}+\lambda x_{0}, x_{2}-\mu x_{0} \in X_{1} \cap C(\lambda, \mu>0)$, then set $\alpha=\frac{\lambda}{\lambda+\mu}, \beta=1-\alpha=\frac{\mu}{\lambda+\mu}$. Since core $C$ 
and $X_{0}$ are convex as well as $\alpha+\beta=1, \alpha, \beta>0$, we have

$$
\alpha\left(x_{2}-\mu x_{0}\right)+\beta\left(x_{1}+\lambda x_{0}\right)=\alpha x_{2}+\beta x_{1}+(\lambda \beta-\mu \alpha) x_{0}=\alpha x_{2}+\beta x_{1} \in X_{0} \cap C .
$$

And then,

$$
F\left(\alpha x_{2}+\beta x_{1}\right)-h\left(\alpha x_{2}+\beta x_{1}\right) \subset K .
$$

That is,

$$
F\left(\alpha\left(x_{2}-\mu x_{0}\right)+\beta\left(x_{1}+\lambda x_{0}\right)\right)-h\left(\alpha x_{2}+\beta x_{1}\right) \subset K .
$$

By the affine property of $h$, we have

$$
\alpha h\left(x_{2}\right)+\beta h\left(x_{1}\right)=h\left(\alpha x_{2}+\beta x_{1}\right) .
$$

By the $K$-convexity of $F$, for all $\lambda, \mu>0$, since $x_{1}+\lambda x_{0}, x_{2}-\mu x_{0} \in X_{1} \cap C$, then

$$
\alpha F\left(x_{2}-\mu x_{0}\right)+\beta F\left(x_{1}+\lambda x_{0}\right) \subset F\left(\alpha\left(x_{2}-\mu x_{0}\right)+\beta\left(x_{1}+\lambda x_{0}\right)\right)+K .
$$

By (6),(7) and (8), we have,

$$
\alpha F\left(x_{2}-\mu x_{0}\right)+\beta F\left(x_{1}+\lambda x_{0}\right)-\alpha h\left(x_{2}\right)-\beta h\left(x_{1}\right) \subset K .
$$

Hence for all $\lambda, \mu>0$, if $x_{1}+\lambda x_{0}, \quad x_{2}-\mu x_{0} \in X_{1} \cap C$, then

$$
\frac{F\left(x_{1}+\lambda x_{0}\right)-h\left(x_{1}\right)}{\lambda}-\frac{h\left(x_{2}\right)-F\left(x_{2}-\mu x_{0}\right)}{\mu} \subset K .
$$

For some $x_{1} \in X_{0}$ and $\lambda>0$ satisfying $x_{1}+\lambda x_{0}$ in $X_{1} \cap C$, taking $y_{1} \in F\left(x_{1}+\lambda x_{0}\right)$, then, $\frac{y_{1}-h\left(x_{1}\right)}{\lambda}$ is an upper bound of the set $\left\{\frac{h(x)-F\left(x-\mu x_{0}\right)}{\mu} \mid x-\mu x_{0} \in X_{1} \cap C, \mu>0\right\}$. Since $Y$ is an order-complete linear space, there exists

$$
y^{S} \equiv \sup \left\{\frac{h(x)-F\left(x-\mu x_{0}\right)}{\mu} \mid x-\mu x_{0} \in X_{1} \cap C, \mu>0\right\},
$$

Similarly, by Remark 2.1, there exists

$$
y^{I} \equiv \inf \left\{\frac{F\left(x+\lambda x_{0}\right)-h(x)}{\lambda} \mid x+\lambda x_{0} \in X_{1} \cap C, \lambda>0\right\} .
$$

Since $y^{S} \leq y^{I}$, then $\left(y^{S}+K\right) \cap\left(y^{I}-K\right)$ is a nonempty set. Taking $\bar{y} \in\left(y^{S}+K\right) \cap\left(y^{I}-K\right)$, then we have

$$
\begin{aligned}
& \frac{F\left(x+\lambda x_{0}\right)-h(x)}{\lambda}-\bar{y} \subset K, \text { if } \lambda>0, x+\lambda x_{0} \in X_{1} \cap C . \\
& \bar{y}-\frac{h(x)-F\left(x-\mu x_{0}\right)}{\mu} \subset K \text {, if } \mu>0, x-\mu x_{0} \in X_{1} \cap C .
\end{aligned}
$$


By (9),

$$
F\left(x+\lambda x_{0}\right)-[h(x)+\lambda \bar{y}] \subset K, \text { if } \lambda>0, \quad x+\lambda x_{0} \in X_{1} \cap C .
$$

By (10),

$$
F\left(x-\mu x_{0}\right)-[h(x)-\mu \bar{y}] \subset K, \text { if } \mu>0, x-\mu x_{0} \in X_{1} \cap C .
$$

We may relabel $-\mu$ by $\lambda$, then

$$
F\left(x+\lambda x_{0}\right)-[h(x)+\lambda \bar{y}] \subset K, \text { if } \lambda<0, x+\lambda x_{0} \in X_{1} \cap C .
$$

Define a map $h_{1}$ from $X_{1}$ to $Y$ as

$$
h_{1}\left(x+\lambda x_{0}\right)=h(x)+\lambda \bar{y}, \forall x+\lambda x_{0} \in X_{1} \cap C .
$$

Then $h_{1}(x)=h(x), \forall x \in X_{0}$. i.e., $h_{1}$ is an extension of $h$ to $X_{1}$. Since $h$ is an affine map, so is $h_{1}$.

From (11) and (12), we know that $h_{1}$ satisfies

$$
F\left(x+\lambda x_{0}\right)-h_{1}\left(x+\lambda x_{0}\right) \subset K, \quad \forall x+\lambda x_{0} \in X_{1} \cap C .
$$

That is,

$$
F(y)-h_{1}(y) \subset K, \quad \forall y \in X_{1} \cap C .
$$

Let $\Gamma$ be the collection of all ordered pairs $\left(X_{\Delta}, h_{\Delta}\right)$, where $X_{\Delta}$ is a subspace of $X$ that contains $X_{0}$ and $h_{\Delta}$ is an affine map from $X_{\Delta}$ to $Y$ that extends $h_{0}$ and satisfies $F(x)-$ $h_{\Delta}(x) \subset K$ for all $x \in X_{\Delta} \cap C$.

Introduce a partial ordering in $\Gamma$ as follows: $\left(X_{\Delta_{1}}, h_{\Delta_{1}}\right) \prec\left(X_{\Delta_{2}}, h_{\Delta_{2}}\right)$ if and only if $X_{\Delta_{1}} \subset X_{\Delta_{2}}, h_{\Delta_{2}}(x)=h_{\Delta_{1}}(x)$ for all $x \in X_{\Delta_{1}}$. If we can show that every totally ordered subset of $\Gamma$ has an upper bound, it will follow from Zorn's Lemma that $\Gamma$ has a maximal element $\left(X_{\max }, h_{\max }\right)$. We can claim that $h_{\max }$ is the desired map. In fact, we must have $X_{\max }=X$. For otherwise, we have shown in the previous proof of this theorem that there would be an $\left(\tilde{X}_{\max }, \tilde{h}_{\max }\right) \in \Gamma$ such that $\left(\tilde{X}_{\max }, \tilde{h}_{\max }\right) \succ\left(X_{\max }, h_{\max }\right)$ and $\left(\tilde{X}_{\max }, \tilde{h}_{\max }\right) \neq\left(X_{\max }, h_{\max }\right)$, This would be violate the maximality of the $\left(X_{\max }, h_{\max }\right)$.

Therefore, it remains to show that every totally ordered subset of $\Gamma$ has an upper bound. Let $M$ be a totally ordered subset of $\Gamma$. Define an ordered pair $\left(X_{M}, h_{M}\right)$ by

$$
X_{M}=\bigcup_{\left(X_{\Delta}, h_{\Delta}\right) \in M}\left\{X_{\Delta}\right\}
$$

And

$$
h_{M}(x)=h_{\Delta}(x), \forall x \in X_{\Delta} \text {, where }\left(X_{\Delta}, h_{\Delta}\right) \in M .
$$


This definition is not ambiguous, for if $\left(X_{\Delta_{1}}, h_{\Delta_{1}}\right)$ and $\left(X_{\Delta_{2}}, h_{\Delta_{2}}\right)$ are any elements of $M$, then either $\left(X_{\Delta_{1}}, h_{\Delta_{1}}\right) \prec\left(X_{\Delta_{2}}, h_{\Delta_{2}}\right)$ or $\left(X_{\Delta_{2}}, h_{\Delta_{2}}\right) \prec\left(X_{\Delta_{1}}, h_{\Delta_{1}}\right)$. At any rate, if $x \in X_{\Delta_{1}} \cap X_{\Delta_{1}}$, then $h_{\Delta_{1}}(x)=h_{\Delta_{2}}(x)$. Clearly, $\left(X_{M}, h_{M}\right) \in \Gamma$. Hence, it is an upper bound for $M$, and the proof is complete.

From Theorem 3.1, we can obtain several interesting results for $K$-convex set-valued maps as follows.

Corollary 3.2. Let $X$ be a real linear space and let $(Y, K)$ be a real order-complete linear topological space, with order cone $K$. Let $F: C \rightarrow 2^{Y}$ be a strictly $K$-convex setvalued map, where $C \subset X$ be a convex set, with $0 \in \operatorname{core} C$. If there exists $y_{0} \in Y$ such that $F(0)-y_{0} \subset K$, then i) there exists an affine map $l: X \rightarrow Y$ such that $l(0)=y_{0}$, and ii) $F(x)-l(x) \subset K$ for all $x \in C$.

Corollary 3.3. Let $X$ be a real linear space and let $(Y, K)$ be a real order-complete linear topological space, with order cone $K$. Let $F: C \rightarrow 2^{Y}$ be a strictly $K$-convex setvalued map, where $C \subset X$ be a convex set, with $x_{0} \in$ core $C$. If there exists $y_{0} \in Y$ such that $F\left(x_{0}\right)-y_{0} \subset K$, then i) there exists an affine map $l: X \rightarrow Y$ such that $l\left(x_{0}\right)=y_{0}$, and ii) $F(x)-l(x) \subset K$ for all $x \in C$.

Proof. Let $D=C-x_{0}$, then $D$ is a convex set and $0 \in$ core $D$. Let

$$
\bar{F}(x)=F\left(x+x_{0}\right),
$$

then $\bar{F}$ is a strictly $K$-convex set-valued map on $D$. And there exists $y_{0} \in Y$ such that $\bar{F}(0)-$ $y_{0} \subset K$. From Corollary 3.2, there exists an affine map $l_{1}: X \rightarrow Y$ such that $l_{1}(0)=y_{0}$, and for all $x \in D, \bar{F}(x)-l_{1}(x) \subset K$. Hence, $l\left(x_{0}\right)=y_{0}$, and for all $x \in C, F(x)-l(x) \subset K$, where $l(x)=l_{1}\left(x-x_{0}\right)$ is an affine map.

Corollary 3.4. Let $X$ be a real linear space and let $(Y, K)$ be a real order-complete linear topological space. Let $F: C \rightarrow 2^{Y}$ be a strictly $K$-convex set-valued map, where $C \subset X$ be a convex set. Let $X_{0}$ be a proper subspace of $X$, with $X_{0} \cap$ core $C \neq \emptyset$. If there is a linear map $T_{0}: X_{0} \rightarrow Y$ such that $F(x)-T_{0}(x) \subset K$ for all $x \in X_{0} \cap C$, then i) there exists a linear map $T: X \rightarrow Y$ such that $T(x)=T_{0}(x)$ for all $x \in X_{0} \cap C$, and ii) $F(x)-T(x) \subset K$ for all $x \in C$.

Proof. By Theorem 3.1, we know that there exists an affine map $l: X \rightarrow Y$ such that $l(x)=T_{0}(x)$, for all $x \in X_{0} \cap C$, and $F(x)-l(x) \subset K$, for all $x \in C$. Suppose that 
$l(x)=T(x)+b$, where $T$ is a linear map from $X$ to $Y$ and $b \in Y$. Since $0 \in X_{0}$ and $T_{0}$ is a linear map, then $b=T(0)+b=l(0)=T_{0}(0)=0$. Hence, $l(x)=T(x)$, for all $x \in C$, and $T(x)=T_{0}(x)$, for all $x \in X_{0} \cap C$. Hence, the conclusion holds.

Let the set-valued map $F: C \rightarrow 2^{Y}$ be replaced by the single-valued map $f: C \rightarrow Y$, then by Corollary 3.4, we have

Corollary 3.5. Let $X$ be a real linear space, and $(Y, K)$ be a real order-complete linear topological space. Let $f: C \rightarrow Y$ be a $K$-convex vector-valued map, where $C \subset X$ be a convex set. Let $X_{0}$ be a proper subspace of $X$, with $X_{0} \cap$ core $C \neq \emptyset$. If there is a linear map $T_{0}: X_{0} \rightarrow Y$ such that $f(x)-T_{0}(x) \in K$ for all $x \in X_{0} \cap C$, then i) there exists a linear map $T: X \rightarrow Y$ such that $T(x)=T_{0}(x)$ for all $x \in X_{0} \cap C$, and ii) $f(x)-T(x) \in K$ for all $x \in C$.

Let $K=[0, \infty)$ and the set-valued map $F: C \rightarrow 2^{Y}$ be replaced by a real-valued function $f: C \rightarrow R$, then by Corollary 3.4, we have

Corollary 3.6. Let $X$ be a real linear space and let $f: C \rightarrow R$ be a convex function, where $C \subset X$ be a convex set. Let $X_{0}$ be a proper subspace of $X$, with $X_{0} \cap$ core $C \neq \emptyset$. If there is a linear functional $T_{0}: X_{0} \rightarrow R$ such that $f(x) \geq T_{0}(x)$ for all $x \in X_{0} \cap C$, then i) there exists a linear functional $T: X \rightarrow R$ such that $T(x)=T_{0}(x)$ for all $x \in X_{0} \cap C$, and ii) $f(x) \geq T(x)$ for all $x \in C$.

Corollary 3.7. Let $X$ be a real linear space and let $(Y, K)$ be a real order-complete linear topological space, with order cone $K$. Let $F: C \rightarrow 2^{Y}$ be a strictly $K$-convex setvalued map, where $C \subset X$ be a convex set, with $0 \in$ core $C$. If $F(0) \subset K$, then i) there exists an linear map $T: X \rightarrow Y$ such that $F(x)-T(x) \subset K$ for all $x \in C$.

Proof. By Corollary 3.2, there exists an affine map $l: X \rightarrow Y$ such that $l(0)=0$, $F(x)-l(x) \subset K$ for all $x \in C$. Assume $l(x)=T(x)+b, \forall x \in X$, where $T$ is a linear map and $b \in Y$. Then $l(0)=T(0)+b=b=0$, and we know that $l=T$, the conclusion holds.

Let $C=X$ and by Corollary 3.7, we have

Corollary 3.8. Let $X$ be a real linear space and let $(Y, K)$ be a real order-complete linear topological space, with order cone $K$. Let $F: X \rightarrow 2^{Y}$ be a strictly $K$-convex set-valued map. If $F(0) \subset K$, then i) there exists an linear map $T: X \rightarrow Y$ such that $F(x)-T(x) \subset K$ for all $x \in X$. 
If the set-valued map $F$ be replaced by a single-valued map $f: X \rightarrow Y$, then by Corollary 3.8 , we have

Corollary 3.9. Let $X$ be a real linear space and let $(Y, K)$ be a real order-complete linear topological space, with order cone $K$. Let $f: X \rightarrow Y$ be a $K$-convex vetor-valued map. If $f(0) \in K$, then i) there exists an linear map $T: X \rightarrow Y$ such that $f(x)-T(x) \in K$ for all $x \in X$.

By Lemma 2.1.2 in [31], a set-valued map $F: C \rightarrow 2^{Y}$ is convex if and only if for every $x, y \in C$ and $\lambda \in(0,1)$

$$
\lambda F(x)+(1-\lambda) F(y) \subset F(\lambda x+(1-\lambda) y) .
$$

Hence, By Theorem 3.1, Corollary 3.4 and Corollary 3.7, respectively, we have some new Hahn-Banach Theorems for a convex set-valued map as follows

Corollary 3.10. Let $X$ be a real linear space, and let $(Y, K)$ be a real order-complete linear topological space. Let the set-valued map $F: C \rightarrow 2^{Y}$ be strictly convex, where $C \subset X$ is a convex set. Let $X_{0}$ be a real linear proper subspace of $X$, with $X_{0} \cap$ core $C \neq \emptyset$ and $h: X_{0} \rightarrow Y$ be an affine map satisfying $F(x)-h(x) \subset K$ for all $x \in X_{0} \cap C$. Then there exists an affine map $l: X \rightarrow Y$ such that i) $l$ is an extension of $h$. i.e., $l(x)=h(x)$ for all $x \in X_{0}$, and ii) $F(x)-l(x) \subset K$ for all $x \in C$.

Corollary 3.11. Let $X$ be a real linear space and let $(Y, K)$ be a real order-complete linear topological space. Let the set-valued map $F: C \rightarrow 2^{Y}$ be strictly convex, where $C \subset X$ be a convex set. Let $X_{0}$ be a proper subspace of $X$, with $X_{0} \cap$ core $C \neq \emptyset$. If there is a linear map $T_{0}: X_{0} \rightarrow Y$ such that $F(x)-T_{0}(x) \subset K$ for all $x \in X_{0} \cap C$, then i) there exists a linear map $T: X \rightarrow Y$ such that $T(x)=T_{0}(x)$ for all $x \in X_{0} \cap C$, and ii) $F(x)-T(x) \subset K$ for all $x \in C$.

Corollary 3.12. Let $X$ be a real linear space and let $(Y, K)$ be a real order-complete linear topological space, with order cone $K$. Let the set-valued map $F: C \rightarrow 2^{Y}$ be strictly convex, where $C \subset X$ be a convex set, with $0 \in$ core $C$. If $F(0) \subset K$, then there exists an linear map $T: X \rightarrow Y$ such that $F(x)-T(x) \subset K$ for all $x \in C$.

If $C \equiv X$, then by Corollary 3.10, we have

Corollary 3.13. Let $X$ be a real linear space and let $(Y, K)$ be a real order-complete linear topological space, with order cone $K$. Let the set-valued map $F: X \rightarrow 2^{Y}$ be strictly convex. If $F(0) \subset K$, then there exists an linear map $T: X \rightarrow Y$ such that $F(x)-T(x) \subset K$ 
for all $x \in X$.

Remark 3.1.(a) Theorem 3.1 and Corollary 3.3, respectively, generalize Theorem 2 and Theorem 3 in [14] from vector-valued to set-valued case. Corollary 3.4 generalized Theorem 2 in [13] from vector-valued to set-valued case.

(b) Let $C=X$, then Corollary 3.6 reduces to Theorem 1.2. Hence, Corollary 3.4, Corollary 3.5 and Corollary 3.6 are all generalizations of Theorem 1.2.

(c) If the set-valued map $F$ is replaced by a single-valued map $p: X \rightarrow Y$, then Corollary 3.7 reduces to Corollary 2.6 in [10].

(d) If the vector-valued map $f$ is replaced by a sublinear map $g: X \rightarrow Y$, then by Corollary 3.9, it is easy to get the Hahn-Banach Theorem in [8, P.18] (i.e., Theorem 3.13 in [6, P.67]) and Corollary in [13, P. 433].

(e) Corollary 3.5 is equivalent to Theorem 2.1 in [10].

(f) It is easy to see that Theorem 3.1 generalizes and unifies Theorem 1.1, Theorem 1.2, Theorem 1.3 and many other results in the literature.

\section{Existence of Subgradients}

The definitions of several kind of subgradients for set-valued maps are necessary be repeated here.

Definition 4.1([27]). Assume that $C \subset X$ and $T \in L(X, Y) . T$ is called a Chen-weak subgradient of $F$ at $x_{0} \in C$ if

$$
F(x)-F\left(x_{0}\right)-T\left(x-x_{0}\right) \subset W, \quad \forall x \in C
$$

where $W=Y \backslash(-i n t K)$. The Chen-weak subdifferential of $F$ at $x_{0}$ is denoted by the set $\partial^{C-W} F\left(x_{0}\right)$ which is all Chen-weak subgradents of $F$ at $x_{0}$.

Definition 4.2([15]). Let $F: C \subset X \rightarrow 2^{Y}$ be $K$-convex, $x_{0} \in C$ and

$$
F\left(x_{0}\right) \cap\left(y_{0}-i n t K\right)=\emptyset,
$$

and $T \in L(X, Y)$ is called a Yang-weak subgradient of $F$ at $\left(x_{0}, y_{0}\right)$ if for all $x \in C$ and for all $y \in F(x)$, we have

$$
y-y_{0}-T\left(x-x_{0}\right) \notin-i n t K .
$$

The set of all Yang-weak subgradents of $F$ at $\left(x_{0}, y_{0}\right)$ is denoted by $\partial_{y_{0}}^{Y-W} F\left(x_{0}\right)$. And we call it to be the Yang-weak subdifferential of $F$ at $\left(x_{0}, y_{0}\right)$. 
Definition 4.3([28]). Let $F: C \subset X \rightarrow 2^{Y}$ be $K$-convex, $x_{0} \in C$ and $F\left(x_{0}\right)-y_{0} \subset K$, $T \in L(X, Y)$ is called a Borwein-strong subgradient of $F$ at $\left(x_{0}, y_{0}\right)$ if for all $x \in C$ and for all $y \in F(x)$, we have

$$
y-y_{0}-T\left(x-x_{0}\right) \in K \text {. }
$$

The set of all Borwein-strong subgradents of $F$ at $\left(x_{0}, y_{0}\right)$ is denoted by $\partial_{y_{0}}^{B-S} F\left(x_{0}\right)$. And we call it to be the Borwein-strong subdifferential of $F$ at $\left(x_{0}, y_{0}\right)$.

Remark 4.1. If $F$ is a single valued map and $y_{0}=F\left(x_{0}\right)$, then the Borwein-strong subgradient reduces to the strong subgradient of vector-valued functions in [14]. And if $F$ is a single valued map and $y_{0}=F\left(x_{0}\right)$, both the Yang-weak subgradient and the Chen-weak subgradient of set-valued maps are consistent with the weak subgradient of vector-valued functions in [14].

Remark 4.2. It is clear that for all $\left(x_{0}, y_{0}\right)$ with $F\left(x_{0}\right)-y_{0} \subset K, \partial_{y_{0}}^{B-S} F\left(x_{0}\right) \subset$ $\partial_{y_{0}}^{Y-W} F\left(x_{0}\right)$.

Remark 4.3. It is clear that if $y_{0} \in F\left(x_{0}\right)$ and $F\left(x_{0}\right) \cap\left(y_{0}-\right.$ int $\left.K\right)=\emptyset$, then $\partial^{C-W} F\left(x_{0}\right) \subset \partial_{y_{0}}^{Y-W} F\left(x_{0}\right)$.

Example. This example shows that $\partial^{Y-W} F\left(x_{0}\right) \not \subset \partial_{y_{0}}^{C-W} F\left(x_{0}\right)$ even if $y_{0} \in F\left(x_{0}\right)$ and $F\left(x_{0}\right) \cap\left(y_{0}-i n t K\right)=\emptyset$ hold. Define a set-valued map $F$ by, $F(x)=\left\{(u, v) \in R^{2}\right.$ : $-1 \leq u \leq 1+|x|,-1 \leq v \leq 1+|x|\}, \quad \forall x \in C=\{x \in R:-1 \leq x \leq 1\} . \quad$ Let $K=\left\{(x, y) \in R^{2}: x \geq 0, y \geq 0\right\}$ and $T \equiv 0: R \rightarrow R^{2}, T(x)=(0,0), \forall x \in R$. Then there exists $x_{0}=0 \in C$ and $z_{0}=(-1,-1) \in F\left(x_{0}\right), F\left(x_{0}\right)-z_{0} \subset K$, for all $x \in C$ and for all $z \in F(x)$, we have:

$$
z-z_{0}-T\left(x-x_{0}\right) \in K \text {. }
$$

Hence $T=0$ is a Borwein-strong subgradient of $F$ at $\left(x_{0}, z_{0}\right)$, and by Remark 4.1, $T=0$ is also a Yang-weak subgradient of $F$ at $\left(x_{0}, z_{0}\right)$. Let $x_{1}=(1,1)$, taking $z_{0}=(1,1) \in F\left(x_{0}\right)$ and $z_{1}=(-1,-1) \in F\left(x_{1}\right)$, then

$$
z_{1}-z_{0}-T\left(x_{1}-x_{0}\right) \in-i n t K .
$$

Hence $T=0$ is not a Chen-weak subgradient of $F$ at $x_{0}$.

Definition 4.4([27]). A subset $S$ of $Y$ is said to be minorized, if there is a $y \in Y$ so that $S \subset y+K$.

we will prove the existence of Yang-weak subgradients of set-valued maps as follows.

Theorem 4.1. Let $X$ be a real linear topological space, and let $(Y, K)$ be a real order linear topological space, with order cone $K$. Let $K$ has a nonempty interior int $K$, Let 
$C \subset X$ be a convex set, with $\operatorname{int} C \neq \emptyset$, let $x_{0} \in \operatorname{int} C$ be given, let $F: X \rightarrow 2^{Y}$ be $K$-convex on $C$ and upper semicontinuous at $x_{0}$, let $-F\left(x_{0}\right)$ be minorized, and there exists $y_{0} \in F\left(x_{0}\right)$ such that

$$
F\left(x_{0}\right) \cap\left(y_{0}-i n t K\right)=\emptyset .
$$

Then there is a Yang-weak subgradient $T$ of $F$ at $x_{0}$ satisfying for every $x \in C$ the property

$$
T\left(x-x_{0}\right) \notin-i n t K \quad \Leftrightarrow \quad T\left(x-x_{0}\right) \in K .
$$

Proof. We define the set $D=C-x_{0}$ and the set-valued map $H: D \rightarrow 2^{Y}$ with $H(x)=F\left(x+x_{0}\right)-y_{0}, \quad \forall x \in D$. Then, $0 \in \operatorname{int} D, D$ is convex, $H$ is upper semicontinuous at $0,0 \in H(0)$, and $-H(0)$ is minorized. In order to see that $H$ is $K$-convex on $D$, take arbitrary $x_{1}, x_{2} \in D$ and $\lambda \in(0,1)$. It follows with the $K$-convexity of $F$

$$
\begin{gathered}
\lambda H\left(x_{1}\right)+(1-\lambda) H\left(x_{2}\right)=\lambda F\left(x_{1}+x_{0}\right)+(1-\lambda) F\left(x_{2}+x_{0}\right)-\lambda y_{0}-(1-\lambda) y_{0} \\
\subset F\left(\lambda x_{1}+(1-\lambda) x_{2}+x_{0}\right)+K-y_{0}=H\left(\lambda x_{1}+(1-\lambda) x_{2}\right)+K .
\end{gathered}
$$

Next we set $P:=\{(x, y) \in X \times Y: x \in D, y \in H(x)+i n t K\}$. By Lemma 2 in [27] and Remark 3 in [27], we obtain $\operatorname{int} P \neq \emptyset$. Now we show that $(0,0) \notin P$. Suppose that $(0,0) \in P$, then there is a $y \in H(0)$ so that $0 \in y+i n t K$ which implies $H(0) \cap(-i n t K) \neq \emptyset$, i.e. $\left(F\left(x_{0}\right)-y_{0}\right) \cap(-i n t K) \neq \emptyset$, this is a contradiction to (16). By Eidelheit's separation theorem for convex sets there is a nonzero $(-\rho, \sigma) \in X^{*} \times Y^{*}$ so that

$$
-\rho(x)+\sigma(y) \geq 0, \quad \forall(x, y) \in P,
$$

where $X^{*}$ and $Y^{*}$, respective, are the topological dual space of $X$ and $Y$.

If $\sigma=0$, then $-\rho(x) \geq 0, x \in D$. Because of $0 \in$ int $D$ we obtain $\rho=0$ contradicting $(-\rho, \sigma) \neq(0,0)$. Hence we get $\sigma \neq 0$. From (17), we get $\sigma(y) \geq 0, \forall(0, y) \in P$. Since

$$
(0, y) \in P \Leftrightarrow y \in H(0)+i n t K \Leftrightarrow \forall \hat{y} \in H(0), y \in \hat{y}+i n t K .
$$

Taking $\hat{y}=0 \in H(0)$, we get $\sigma(y) \geq 0, \forall y \in$ int $K$. Hence $\sigma(y) \geq 0, \forall y \in K$, that is $\sigma \in K^{*}$ ( the polar cone of $K$ ). Then there is a $\bar{y} \in$ int $K$ with $\sigma(\bar{y})=1$. We now define a map $T: X \rightarrow Y$ by $T(x)=\rho(x) \bar{y}, \forall x \in X$. Obviously, $T$ is linear and continuous. Next we prove that for all $x \in C$ and for all $y \in F(x)$,

$$
y-y_{0}-T\left(x-x_{0}\right) \notin-i n t K
$$

or for all $x \in D$ and for all $y \in H(x)$,

$$
y-T(x) \notin-i n t K .
$$


Suppose that there is some $x \in D$ and some $y \in H(x)$ with $y-T(x) \in-i n t K$. Because of $\sigma \in K^{*} \backslash\{0\}$ we then get $0>\sigma(y-T(x))=\sigma(y)-\rho(x) \sigma(\bar{y})=\sigma(y)-\rho(x)$. This is a contradiction to the inequality (17). Hence, the condition (18) is fulfilled and, therefore, $T$ is a Yang-weak subgradient at $\left(x_{0}, y_{0}\right)$. Finally, for every $x \in D$ we get

$T(x) \notin-i n t K \quad \Leftrightarrow \quad \rho(x) \bar{y} \notin-i n t K \quad \Leftrightarrow \quad \rho(x) \geq 0 \quad \Leftrightarrow \quad T(x) \in K$.

Remark 4.4.(a) Theorem 4.1 is the existence theorem of Yang-weak subgradient. The formula (16) is weaker than the condition $F\left(x_{0}\right) \cap\left(F\left(x_{0}\right)-i n t K\right)=\emptyset$ which is rather restrictive for the set $F\left(x_{0}\right)$. Hence this result improves Theorem 7 in [27].

(b)Let $X$ be a real linear topological space, and let $(Y, K)$ be a real order linear topological space, where $K$ has a nonempty interior int $K$. Let the vector-valued map $f: C \rightarrow Y$ be $K$-convex on $C$ and continuous at $x_{0} \in \operatorname{int} C$, where $C \subset X$ be a convex set with $\operatorname{int} C \neq \emptyset$. By Theorem 4.1, if $y_{0}=f\left(x_{0}\right)$, then there is a weak subgradient $T$ of $f$ at $x_{0}$ satisfying for every $x \in C$ the property

$$
T\left(x-x_{0}\right) \notin-i n t K \quad \Leftrightarrow \quad T\left(x-x_{0}\right) \in K .
$$

This is Theorem 1 in [14]. Hence, Theorem 4.1 is also a generalization of Theorem 1 in [14].

By using the Hahn-Banach theorem which we obtained in section 3, the existence of Borwein-strong subgradient of set-valued maps can also be obtained.

Theorem 4.2. Let $X$ be a real linear space and let $(Y, K)$ be a real order-complete linear topological space. Let $F: C \rightarrow 2^{Y}$ be a strictly $K$-convex set-valued map, where $C$ is a convex set with $x_{0} \in \operatorname{core} C$. If there exists $y_{0} \in Y$ such that $F\left(x_{0}\right)-y_{0} \subset K$, then there exists a Borwein-strong subgradient of $F$ at $\left(x_{0}, y_{0}\right)$.

Proof. By Corollary 3.3, there exists an affine map $l: X \rightarrow Y$ such that $l\left(x_{0}\right)=y_{0}$, and for all $x \in C, F(x)-l(x) \in K$. Then for some $T \in L(X, Y)$.

$$
l(x)=l\left(x_{0}\right)+T\left(x-x_{0}\right), \forall x \in X .
$$

and

$$
F(x)-l\left(x_{0}\right)-T\left(x-x_{0}\right) \subset K, \forall x \in X .
$$

that is,

$$
F(x)-y_{0}-T\left(x-x_{0}\right) \subset K, \forall x \in X .
$$

Remark 4.5. Let $C$ be a convex set in $X$, and let $(Y, K)$ be a real order-complete linear topological space. If the vector-valued map $f: C \rightarrow Y$ be $K$-convex and $x_{0} \in$ core $C$, then 
by Theorem 4.2 ,

$$
\partial_{y_{0}}^{S} f\left(x_{0}\right)=\left\{T \in L(X, Y) \mid f(x)-y_{0}-T\left(x-x_{0}\right) \in K, \forall x \in C\right\} \neq \emptyset,
$$

for any $f\left(x_{0}\right)-y_{0} \in K$. Particularly, let $y_{0}=f\left(x_{0}\right)$,

$$
\partial^{S} f\left(x_{0}\right)=\left\{T \in L(X, Y) \mid f(x)-f\left(x_{0}\right)-T\left(x-x_{0}\right) \in K, \forall x \in C\right\} \neq \emptyset .
$$

This is Theorem 4 in [14]. Hence, Theorem 4.2 is a generalization of Theorem 4 in [14].

\section{The Lagrange Multiplier Theorem}

Some Lagrange multiplier theorems have been proven in [33], [34] and [15]. In this section, by using the Hahn-Banach extension theorem which we obtained in section 3, a new Lagrange multiplier theorem is obtained. Then we apply this result to derive the existence for strong Lagrange multiplier of equality constraint vector optimization problem.

Let $F: X \rightarrow 2^{Y}, H: X \rightarrow 2^{Z}$ be set-valued maps. The set-valued map $M: Z \rightarrow 2^{Y}$ is defined by $M(z)=F \circ H^{-1}(z)=\{F(x): z \in H(x)\}$. We consider the program

$$
\text { Strong }-\operatorname{Min}\{F(x) \mid 0 \in H(x)\}
$$

The point $\mu \in Y$ is a strong minimum of $(\mathrm{P})$, if there exists $x_{0} \in X$, such that $0 \in H(x)$, $\mu \in F\left(x_{0}\right)$ and $y-\mu \in K, \forall y \in F(x), 0 \in H(x)$.

Theorem 5.1. Consider the problem (P). If the set-valued map $M=F \circ H^{-1}$ is strictly $K$-convex on $Z$, then for any strong minimum $\mu$ of $(\mathrm{P})$, there exists a linear map $T: Z \rightarrow Y$ such that

$$
F(x)+T \circ H(x)-\mu \subset K, \quad \forall x \in X .
$$

proof. Let $\mu$ be a strong minimum of $(\mathrm{P})$ and $N(z)=M(z)-\mu, \forall z \in Z$, then the set-valued map $N$ is strictly $K$-convex on $Z$. By $M(0)=F \circ H^{-1}(0)=\{F(x): 0 \in H(x)\}$, we have $N(0) \subset K$. By Corollary 3.7, there exists a linear map $T_{1}: Z \rightarrow Y$, such that

$$
N(z)-T_{1}(z) \subset K, \quad \forall z \in Z .
$$

that is,

$$
M(z)-\mu-T_{1}(z) \subset K, \quad \forall z \in Z .
$$


Let $z \in H(x), y \in F(x) \subset F \circ H^{-1}(z)=M(z)$,

$$
y-T_{1}(z)-\mu \subset K,
$$

Let $T=-T_{1}$, we get

$$
F(x)+T \circ H(x)-\mu \subset K .
$$

Example 5.1. Consider the problem:

$$
\text { Strong }-\operatorname{Min}\{F(x) \mid x \in X, A x=b\}
$$

where $F: X \rightarrow 2^{Y}$ is strictly $K$-convex on $X . A: X \rightarrow Z$ is a linear map but isn't a zero linear map, $b \in Z$. Let $H(x)=A x-b, \forall x \in X$. Then (P1) becomes

$$
\text { Strong }-\operatorname{Min}\{F(x) \mid x \in X, 0 \in H(x)\}
$$

The set-valued map $F \circ H^{-1}$ is strictly $K$-convex on $Z$. From Theorem 5.1, for any strong minimum $\mu$ of $(\mathrm{P})$, there exists a linear map $T: Z \rightarrow Y$ such that

$$
F(x)+T \circ H(x)-\mu \subset K, \quad \forall x \in X,
$$

i.e.

$$
F(x)+T \circ(A x-b)-\mu \subset K, \quad \forall x \in X .
$$

\section{Sandwich Theorem}

The sandwich theorem has been researched in [6] and [10]. In this section, we present a sandwich theorem for convex set-valued maps and another new sandwich theorem for $K$-convex vector-valued maps

Theorem 6.1. $\quad$ Let $X$ be a real linear space and let $(Y, K)$ be a real order-complete linear topological space, with order cone $K$. Let the set-valued maps $H_{1}: X \rightarrow 2^{Y}$ and $H_{2}: X \rightarrow 2^{Y}$ be strictly convex, respectively. Suppose that

$$
H_{1}(x)-H_{2}(x) \subset K, \quad \forall x \in X .
$$

Then there exists an affine map $l: X \rightarrow Y$ such that $H_{1}(x)-l(x) \subset K$ and $l(x)-H_{2}(x) \subset K$ for all $x \in X$.

Proof. We define a set-valued map $H: X \rightarrow 2^{Y}$ by $G r(H)=G r\left(H_{1}\right)-G r\left(H_{2}\right)$. Then it is apparent that the set-valued map $H$ is convex. And we can prove that $H$ is strict on $X$. In fact, by the strictness of $H_{1}$ and $H_{2}, \forall x \in X, \exists \bar{y} \in H_{1}(x), \exists y_{0} \in H_{2}(0)$ such that 
$\left(x, \bar{y}-y_{0}\right)=(x, \bar{y})-\left(0, y_{0}\right) \in G r(H)$. That is, $\bar{y}-y_{0} \in H(x), \forall x \in X$. Hence, $H$ is a strict set-valued map on $X$. And we can also prove that

$$
H(0) \subset K .
$$

In fact, for arbitrary $y \in H(0)$, i.e., $(0, y) \in G r(H)=G r\left(H_{1}\right)-G r\left(H_{2}\right)$, there exist $\left(x_{1}, y_{1}\right) \in \operatorname{Gr}\left(H_{1}\right)$ and $\left(x_{2}, y_{2}\right) \in \operatorname{Gr}\left(H_{2}\right)$ such that $(0, y)=\left(x_{1}, y_{1}\right)-\left(x_{2}, y_{2}\right)$. Then, $x_{1}=x_{2}=x \in X$ and $y=y_{1}-y_{2} \in H_{1}(x)-H_{2}(x) \subset K$. Hence, $H(0) \subset K$.

By Corollary 3.13, there exists a linear map $T: X \rightarrow Y$ such that

$$
H(x)-T(x) \subset K, \forall x \in X .
$$

If $x=x_{1}-x_{2}$, then $H_{1}\left(x_{1}\right)-H_{2}\left(x_{2}\right) \subset H(x)$ and so

$$
H_{1}\left(x_{1}\right)-H_{2}\left(x_{2}\right)-T(x) \subset K
$$

By $T(x)=T\left(x_{1}\right)-T\left(x_{2}\right)$ and (19), we have

$$
H_{1}\left(x_{1}\right)-T\left(x_{1}\right)-\left(H_{2}\left(x_{2}\right)-T\left(x_{2}\right)\right) \subset K .
$$

Since $Y$ is an order-complete linear space, there exist

$$
y^{S} \equiv \sup \left\{H_{2}(x)-T(x) \mid x \in X\right\}
$$

and

$$
y^{I} \equiv \inf \left\{H_{1}(x)-T(x) \mid x \in X\right\} .
$$

Since $y^{S} \leq y^{I}$, then $\left(y^{S}+K\right) \cap\left(y^{I}-K\right)$ is a nonempty set. Taking $\bar{b} \in\left(y^{S}+K\right) \cap\left(y^{I}-K\right)$, then for all $x \in X$,

$$
H_{1}(x)-T(x)-\bar{b} \subset K,
$$

and

$$
\bar{b}+T(x)-H_{2}(x) \subset K .
$$

Define a map $l: X \rightarrow Y$ as $l(x)=T(x)+\bar{b}, \forall x \in X$. Then $l$ is affine and for all $x \in X$, $H_{1}(x)-l(x) \subset K$, and $l(x)-H_{2}(x) \subset K$.

Corollary 6.2. Let $X$ be a real linear space and let $(Y, K)$ be a real order-complete linear topological space, with order cone $K$. Let $h_{1}: X \rightarrow Y$ is $K$-convex vector-valued map, that is, for every $x, y \in X$ and $\lambda \in(0,1)$

$$
\lambda h_{1}(x)+(1-\lambda) h_{1}(y) \in h_{1}(\lambda x+(1-\lambda) y)+K
$$


and $h_{2}: X \rightarrow Y$ is $K$-concave vector-valued map, that is, for every $x, y \in X$ and $\lambda \in(0,1)$

$$
\lambda h_{2}(x)+(1-\lambda) h_{2}(y) \in h_{2}(\lambda x+(1-\lambda) y)-K .
$$

Suppose that

$$
h_{1}(x)-h_{2}(x) \in K, \quad \forall x \in X .
$$

Then there exists an affine map $l: X \rightarrow Y$ such that $h_{1}(x)-l(x) \in K$ and $l(x)-h_{2}(x) \in K$ for all $x \in X$.

Proof. Define two set-valued maps $H_{1}: X \rightarrow 2^{Y}$ and $H_{2}: X \rightarrow 2^{Y}$, respectively, as $H_{1}(x)=h_{1}(x)+K, \forall x \in X$ and $H_{2}(x)=h_{2}(x)-K, \forall x \in X$. It is easy to prove that both $H_{1}$ and $H_{2}$ are strict convex set-valued map. And we have

$$
H_{1}(x)-H_{2}(x)=\left(h_{1}(x)+K\right)-\left(h_{2}(x)-K\right) \subset K, \quad \forall x \in X .
$$

By Theorem 6.1, we know that there exists an affine map $l: X \rightarrow Y$ such that $H_{1}(x)-l(x) \subset$ $K$ and $l(x)-H_{2}(x) \subset K$ for all $x \in X$. Specially, we have $h_{1}(x)-l(x) \in K$ and $l(x)-h_{2}(x) \in K$ for all $x \in X$.

\section{References}

[1] Hahn H (1927) über lineare Gleichungssysteme in linearen Räumen. J. reine und angew. Math. 157: 214-229

[2] Banach S (1932) Théorie des Opérations Linéaires. Warszawa

[3] Bohnenblust HF, Sobczyk A (1938) Extensions of functionals on complex linear spaces, Bull. Amer. Math. Soc. 44:91-93

[4] Weston JD (1960) A note on the extension of linear functionals. Amer. Math. Monthly 67: $444-445$

[5] Hirano N, Komiya H, Takahashi W (1982) A generalization of the Hahn-Banach Theorem. J. Math. Anal. Appl. 88: 333-340

[6] Jahn J. (1986) Mathematical vector optimization in partially ordered linear spaces, Verlag Peter Lang

[7] Day MM (1962) Normed Linear Space. Springer-verlag, Berlin

[8] Zowe J (1976) Konvexe Funktionen und konvexe dualitätstheorie in geordneten vektorräumen, Habilitation Thesis, University of Würzburg

[9] Zowe J (1975) Linear maps majorized by a sublinear map. Arch. Math. 26:637-645

[10] Zowe J (1978) Sandwich theorems for convex operators with values in an order vector space, J. Math. Anal. Appl. 78:282-296 
[11] Elster KH, Nehse R (1978) Necessary and sufficient conditions for order-completeness of partially ordered vector spaces, Math. Nachr. 81:301-311

[12] Wang SS (1986) A separation theorem of convex cone on ordered vector space and its application, Acta Math. Appl. Sinica 9(3): 309-318.(in Chinese)

[13] Shi SZ (1985) A separation theorem of convex sets for a complete vector lattice and its application, Chinese Annals of Mathematics, 6A(4): 431-438.(in Chinese)

[14] Chen GY, Craven BD (1990) A vector variational inequality and Optimization over an efficient set. Methods and Models of Operations Research 34: 1-12

[15] Yang XQ (1992) A Hahn-Banach theorem in ordered linear spaces and its applications, Optimization 25: 1-9

[16] Meng ZQ (1998) Hahn-Banach Theorems of Set-valued map, Applied Mathematics and Mechanics 19(1): 59-66

[17] Eidelheit M (1936) Zur Theorie der konvexen Mengen in linearen normierten Räumen, Studia Math. 6:104-111

[18] Rockafellar RT (1970) Convex analysis, Princeton University Press, Princeton

[19] Deumlich R, Elster KH and Nehse R (1978) Resent results on the separation of convex sets, Math. Operationsforsch . Statist. ser. Optim. 9: 273-294

[20] Taylor AE, Lay DC (1980) Introduction to functional analysis, John Wiley \& Sons

[21] Jahn J (1996) Introduction to the theory of nonlinear optimization, Springer-Verlag, Berlin

[22] Kantorvitch L, Akilov G (1959) Functional analysis in normed spaces, Moscow

[23] Lassonde (1998) Hahn-Banach theorems for convex function, in Ricceri/Simons, Minimax theorems and applications, Kluwer

[24] Rudin W (1973) Functional analysis, McGraw-Hill, Inc.

[25] Schechter M (1971) Principles of functional analysis, Academic Press, Inc.

[26] Aubin JP, Ekeland I (1984) Applied Nonlinear Analisis. John Wiley \&Sons

[27] Chen GY, Jahn J (1998) Optimality conditions for set-valued optimization problems. Mathematical Metheds of Operations Research 48(2): 187-200

[28] Borwein JM (1981)A lagrange multiplier theorem and a sandwich theorem for convex relations, Math. Scand. 48: 189-204

[29] Jameson G (1970) Ordered linear space. Lecture Notes in Mathematics 141. SpringerVerlag

[30] Sawaragi Y, Nakayama H, Tanino T ( 1985) Theory of Multiobjective Optimization. Academic Press INC

[31] Aubin JP, Frankowska H (1990) Set-Valued Analysis, Boston:Birkhaüser

[32] Yosida K (1965) Functional analysis, Springer-verlag 
[33] Corley HW (1981) Duality theorem for maximizations with respect to cones, J. Math. Anal. Appl. 84:560-568

[34] Lai HC, Yang LS (1989) Strong duality for infinite-dimensional vector-valued programming problems, J. Optim. Theory and Appl.62:449-466

[35] Zowe J (1974) Subdifferentiability of convex functions with values in ordered vector spaces, Math. Scand. 34:69-83

[36] Zowe J (1975) A duality theorem for a convex programming programming problem in order complete vector lattices, J. Math. Anal. Appl. 50: 273-287

[37] Elster KH and Nehse R (1978) Separation of two convex sets by operators, Math. Univ. Carolinae (19)(1): 191-201 